\title{
Effect of GEN1 interference on the chemosensitivity of the breast cancer MCF-7 and SKBR3 cell lines
}

\author{
YUNLU WU ${ }^{1,2}$, YING QIAN ${ }^{1}$, GUOZHONG ZHOU ${ }^{1}$, JUAN LV ${ }^{1}$, QIUYUE YAN ${ }^{1,2}$ and XUEJUN DONG ${ }^{1,2}$ \\ ${ }^{1}$ Clinical Laboratory Center, Shaoxing People's Hospital, Shaoxing Hospital of Zhejiang University, Shaoxing, \\ Zhejiang 312000; ${ }^{2}$ The Key Laboratory of Laboratory Medicine, Ministry of Education of China, \\ School of Laboratory Medicine and Life Science, Wenzhou Medical University, \\ Wenzhou, Zhejiang 325000, P.R. China
}

Received January 23, 2015; Accepted March 1, 2016

DOI: $10.3892 / 01.2016 .4489$

\begin{abstract}
Chemotherapy is a notable method for the treatment of breast cancer. Numerous genes associated with the sensitivity of cancer to chemotherapy have been found. In recent years, evidence has suggested that a particular structure termed Holliday junction ( $\mathrm{HJ}$ ) plays a crucial role in cancer chemosensitivity. Targeting HJ resolvases, such as structure-specific endonuclease subunit SLX4 (Slx4) and MUS81 structure-specific endonuclease subunit (Mus81), significantly increases the chemosensitivity of tumor cells. Flap endonuclease GEN homolog 1 (GEN1) is a HJ resolvase that belongs to the Rad2/xeroderma pigmentosum complementation group G nuclease family. Whether GEN1 affects the chemosensitivity of tumor cells in a similar manner to Slx4 and Mus81 remains unknown. The aim of the present study was to determine the effect of GEN1 interference on the chemosensitivity of breast cancer cell lines. The investigation of the function of GEN1 was performed using MCF-7 and SKBR3 cells. Short hairpin RNA was used to suppress the expression of GEN1, and western blot analysis and reverse transcription-quantitative polymerase chain reaction were used to detect gene expression. In addition, a cell counting kit-8 assay was performed to detect the viability of cells and flow cytometry was performed to test apoptosis levels. Suppression of GEN1 in SKBR3 cells effectively increased the sensitivity to the chemotherapeutic drug 5-fluorouracil (5-FU), while MCF-7 cells showed no significant change in sensitivity following GEN1 suppression. However, when GEN1 was targeted in addition to Mus81, the MCF-7 cells also demonstrated a significantly increased sensitivity to 5-FU. In addition, when the level of Mus81 was low, GEN1 expression
\end{abstract}

Correspondence to: Professor Xuejun Dong, Clinical Laboratory Center, Shaoxing People's Hospital, Shaoxing Hospital of Zhejiang University, 568 Zhongxing North Road, Shaoxing, Zhejiang 312000, P.R. China

E-mail: dxj9666@163.com

Key words: GEN1, 5-fluorouracil, chemosensitivity, breast cancer cells, Mus81 was increased under a low concentration of 5-FU. The present results suggest that GEN1 may play different roles in different breast cancer cell lines. The function of GEN1 may be affected by the level of Mus81 in the cell line. In addition, GEN1 interference may improve the sensitivity to chemotherapy induced by targeting Mus 81 alone.

\section{Introduction}

Breast cancer is one of the most common malignant tumors worldwide (1). At present, chemotherapy remains an important method for the treatment of breast cancer. However, chemoresistance has become a non-negligible factor for hindering the prognosis of patients (2). Treatments that increase the chemosensitivity of breast cancer, and therefore increase the patient survival rate, are urgently required.

Flap endonuclease GEN homolog 1 (GEN1) was first proposed by Furukawa et al (3) and belongs to a new class of the $\operatorname{Rad} 2 /$ xeroderma pigmentosum complementation group $G$ (XPG) nuclease family, class IV (4). Previous studies have demonstrated that GEN1 is able to resolve a particular structure termed Holliday junctions (HJs), which are formed during DNA strand exchange, as a central intermediate in the process of homologous recombination (5-7). Numerous studies have hypothesized that resolving HJs properly is the key to correct DNA repair $(8,9)$. DNA damaging drugs, such as 5-fluorouracil (5-FU), injure tumor cells by damaging the DNA of the cells. During this process, numerous HJs are produced. Identifying $\mathrm{HJ}$ resolvases in tumor cells is essential for an improved understanding to tumor self-repair (10). At present, certain enzymes have been identified, including Bloom syndrome, RecQ helicase-like/slow growth suppressor 1, MUS81 structure-specific endonuclease subunit (Mus81)-Mms4/essential meiotic structure-specific endonuclease 1, Rad1-Rad10 and structure-specific endonuclease subunit SLX1-structure-specific endonuclease subunit SLX4 (Slx4) (5,11). Certain evidence has indicated that the ability of tumor cells to process HJ determined the sensitivity of the cells to DNA-damaging drugs (12-14). It has been confirmed that subsequent to suppressing the HJ resolvase Slx4, the sensitivity of tumor cells to DNA damaging agents increased significantly (12). Previous studies also confirmed that targeting Mus81 increases sensitivity to DNA damaging 
drugs, such as 5-FU, in breast cancer cells (13). However, it remains unknown whether GEN1 affects the chemosensitivity of tumor cells, such as Slx4 and Mus81. It has been shown that GEN1 interference increases the pharmaceutical sensitivity of yeast and Drosophila alone or in combination with other genes $(11,15)$. Therefore, the present study aimed to explore the effect of GEN1 interference on the chemosensitivity of breast cancer MCF-7 and SKBR3 cell lines.

\section{Materials and methods}

Reagents. The breast MCF-7 and SKBR3 cell lines were purchased from the Shanghai Cell Bank of Chinese Academy of Sciences (Shanghai, China). HyClone Minimum essential medium (MEM) and Roswell Park Memorial Institute-1640 medium (RPMI-1640) were purchased from GE Healthcare Life Sciences (Logan, UT, USA). Gibco fetal bovine serum (FBS), penicillin and streptomycin were purchased from Thermo Fisher Scientific (Waltham, MA, USA). 5-FU was purchased from Hangzhou Bioer Technology Co.,Ltd. (Hangzhou, China). Lipofectamine 2000 and TRIzol reagent were purchased from Thermo Fisher Scientific. The short hairpin (sh)RNA interference plasmid and the plasmid containing shRNA without RNA interference were purchased from Shanghai Genechem Co., Ltd. (Shanghai, China). The Annexin V-fluorescein isothiocyanate (FITC)/propidium iodide (PI) kit and the Cell Counting kit-8 (CCK-8) were purchased from Nanjing KeyGen Biotech Co., Ltd. (Nanjing, China) for cell apoptosis detection and cell viability assays. For reverse transcription-quantitative polymerase chain reaction (RT-qPCR), First-Strand cDNA Synthesis kit and 2X Taq PCR Mix were purchased from Biomiga (San Diego, CA, USA). The LightCycler 480 PCR apparatus was purchased from Hoffmann-La Roche (Basel, Switzerland). In addition, for western blot analysis, primary antibodies against GEN1 (dilution, 1:75), Mus81 (dilution, $1: 1,000$ ) and $\beta$-actin (dilution, 1:5,000) were purchased from Biorbyt (Cambridge, UK), GeneTex (Irvine, CA, USA) and Abcam (Cambridge, UK), respectively. The secondary antibody against rabbit immunoglobulin ( $\mathrm{Ig}) \mathrm{G}$ conjugated to horseradish peroxidase (dilution, 1:3,000) was purchased from Hangzhou HuaAn Biotechnology Co., Ltd. (Hangzhou, China). The ECL-Plus chemiluminescence detection kit was purchased from Beyotime Institute of Biotechnology (Haimen, China), and the PowerPac HC TRANS-BLOT equipment was purchased from Bio-Rad Laboratories (Hercules, CA, USA). The CKX41 inverted fluorescence microscope was purchased from Olympus Corporation (Tokyo, Japan).

Cell culture. The MCF-7 cells were cultured in MEM and SKBR3 cells were cultured in RPMI-1640. MEM and RPMI-1640 were each supplemented with $10 \%$ FBS, $100 \mathrm{U} / \mathrm{ml}$ penicillin and $100 \mathrm{mg} / \mathrm{ml}$ streptomycin. The cells were cultured at $37^{\circ} \mathrm{C}$ in a $5 \% \mathrm{CO}_{2}$ atmosphere.

shRNA transfection. The MCF-7 and SKBR3 cells were grown in 6-well plates at a density of $1 \times 10^{6}$ cells per well in MEM and RPMI-1640 with $10 \%$ FBS, but without antibiotics, as antibiotics would cause cell damage in the transfection process. When the cells had reached $70-80 \%$ confluency in the antibiotic-free medium, the medium was changed to MEM and RPMI-1640 without FBS or antibiotics, to prepare for transfection. The expression of GEN1 and Mus81 was knocked-down by transfection with an shRNA interference plasmid directed against GEN1 and Mus81, with the addition of $4 \mu \mathrm{g}$ plasmid per well. The plasmid containing shRNA without RNA interference was used as a negative control. Cells were transfected in serum-free and antibiotic-free MEM and RPMI-1640 medium for $6 \mathrm{~h}$. The medium was then changed to complete medium (MEM and RPMI-1640 with $10 \%$ FBS and $1 \%$ antibiotic) to cultivate continuously. Transfection was performed using Lipofectamine 2000, according to the manufacturer's protocol. Interference was determined by RT-qPCR and western blotting. The shRNA sequences were as follows: GEN1 shRNA (sh-GEN1), 5'-CCGGGCAAATTAAAGCTGTCAGTAACT CGAGTTACTGACAGCTTTAATTTGCTTTTTG-3'; Mus81 shRNA (sh-Mus81), 5'-CCGGGAGTTGGTACTGGATCACAT TCTCGAGAATGTGATCCAGTACCAACTCTTTTTG-3'; and control shRNA (sh-Ctrl), 5'-TTTCTCCGAACGTGTCACGTT TCAAGAGAACGTGACACGTTCGGAGAATTTTTTC-3'.

$R T$ - $q P C R$. Cells were grown in 6-well- plates at a density of $1 \times 10^{6}$ cells per well in medium containing $10 \% \mathrm{FBS}$ at $37^{\circ} \mathrm{C}$ in a $5 \% \mathrm{CO}_{2}$ atmosphere. Subsequent to the transfection of cells with interference plasmids containing sh-GEN1 or sh-Mus81, and control plasmid containing sh-Ctrl for $24 \mathrm{~h}$, total RNA was extracted. Total cellular RNA was isolated with TRIzol reagent, followed by RT with the First-Strand cDNA Synthesis kit, according to the manufacturer's protocol. RT-qPCR was performed using the LightCycler 480 PCR apparatus. The abundance of the GEN1 and Mus81 transcripts was expressed relative to the control of $\beta$-actin. The experiments were performed three times independently. The Mus81 and $\beta$-actin primer sequences were previously described (13), while the GEN1 primer sequence was identified from a previous study (16). The primer sequences are listed in Table I.

Western blotting. The cells were harvested and rinsed with phosphate-buffered saline (Solarbio Biotechnology, Beijing, China). Total proteins were extracted using radioimmunoprecipitation assay lysis buffer (Biomiga). The protein concentration was determined using the bicinchoninic acid assay (Beyotime Institute of Biotechnology). Equal amounts of the proteins were separated using $10 \%$ gel electrophoresis and electrophoretically transferred to polyvinylidene difluoride (PVDF) membranes (Mylab China, Beijing, China). The membranes were blocked with $5 \%$ skim milk for $2 \mathrm{~h}$ at room temperature. The PVDF membranes were incubated with primary antibodies against GEN1 (dilution, 1:75), Mus81 (dilution, 1:1,000) and $\beta$-actin (dilution, 1:5,000) overnight at $4^{\circ} \mathrm{C}$, followed by incubation in secondary antibodies against rabbit IgG conjugated to horseradish peroxidase (dilution, 1:3,000) for $1 \mathrm{~h}$ at room temperature. The blots were then developed using the ECL-Plus chemiluminescence detection kit, according to the manufacturer's protocol. Subsequently, the blots were exposed to a radiographic film (Kodak, Rochester, NY, USA). $\beta$-actin expression was used as a control. The gray scale was scanned by ChemiDocTM XRS gel imaging system (Bio-Rad Laboratories) and 'gray value' semi-quantitative analysis was performed using Quantity One-4.6.2 software (Bio-Rad Laboratories). The abundance of the GEN1 and 
Table I. Sequences of the primers used for reverse transcription-quantitative polymerase chain reaction.

\begin{tabular}{llc}
\hline Gene & \multicolumn{1}{c}{ Primer sequence, 5'-3' $^{\prime}$} & Product size, bp \\
\hline $\begin{array}{l}\text { GEN1 } \\
\text { Forward } \\
\text { Reverse }\end{array}$ & CCACATGACTATGAATACTGCTGTCCTT \\
Mus81 & TGGGAATCCCTCACAACAGCAAGC & 117 \\
Forward & TGTGGACATTGGCGAGAC & 319 \\
Reverse & GCTGAGGTTGTGGACGGA & 60 \\
$\beta$-actin & & 108 \\
Forward & ACCCACACTGTGCCCATCTAC & 60 \\
Reverse & TCGGTGAGGATCTTCATGAGGTA & 6 \\
\hline
\end{tabular}

GEN1, flap endonuclease GEN homolog 1; Mus81, MUS81 structure-specific endonuclease subunit; Tm, temperature.

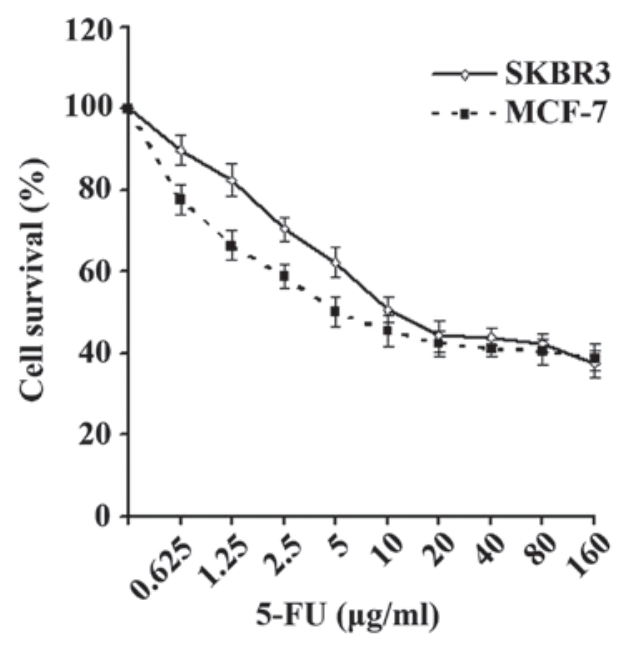

Figure 1. Survival of SKBR3 and MCF-7 cells under various concentrations of 5-FU. Cell survival was determined using an CCK-8 assay. In total, $1 \times 10^{4}$ SKBR3 or MCF-7 cells were planted into 96-well culture plates and various concentrations of 5 -FU were added $24 \mathrm{~h}$ later. Subsequent to the exposure of cells to 5-FU for $48 \mathrm{~h}, 10 \mu \mathrm{l} \mathrm{CCK}-8$ medium was added to each well and the optical density values were determined using a microplate reader $1 \mathrm{~h}$ later. 5-FU, 5-fluorouracil; CCK-8, cell counting kit-8.

Mus81 proteins was expressed relative to the expression of the $\beta$-actin control. Relative gene expression was calculated by normalization to that of $\beta$-actin. The calculation formula was as follows: Relative expression of target gene $=$ Gray value of the target gene / Gray value of $\beta$-actin.

Determination of the half maximal inhibitory concentration $\left(I_{50}\right)$ values for 5-FU in SKBR3 and MCF-7 cells. Cells in a good condition (firmly adherent, clear pseudopodia and clear cytoplasm without impurities) were seeded onto 96 -well plates at a density of $1 \times 10^{4}$ cells per well. Subsequent to $24 \mathrm{~h}$, the cells were incubated with 5 -FU at concentrations ranging between 0.625 and $10 \mu \mathrm{g} / \mathrm{ml}$ for $48 \mathrm{~h}$. Cells not treated with $5-\mathrm{FU}$ were used as a negative control. Finally, $10 \mu$ l water-soluble tetrazolium salt-8 (Nanjing KeyGen Biotech Co., Ltd.) was added into each well. Optical density (OD) values were measured at a wavelength of $450 \mathrm{~nm}\left(\mathrm{OD}_{450}\right)$ using a microplate reader (Anthos 2000; Anthos Labtec Instruments GmbH,
Salzburg, Austria). Cell survival was calculated as follows: Survival of cells $(\%)=$ Drug-treated group $\mathrm{OD}_{450} /$ control group $\mathrm{OD}_{450} \times 100$. The $\mathrm{IC}_{50}$ value was calculated as follows: $\log \mathrm{IC}_{50}=\mathrm{Xm}-\mathrm{I}[\mathrm{P}-(3-\mathrm{Pm}-\mathrm{Pn}) / 4]$, where Xm was the log maximum dose, I was the log (maximum dose/adjacent dose), $\mathrm{P}$ was the sum of the positive response rate, Pm was the maximum positive response rate and $\mathrm{Pn}$ was the minimum positive response rate.

Cell survival assays. Subsequent to transfection with shRNA for $24 \mathrm{~h}$, the cells were planted into 96-well plates at a density of $1 \times 10^{4}$ cells per well. Subsequent to $24 \mathrm{~h}$, the cells were incubated with $5-\mathrm{FU}$ at concentrations ranging between $0.625 \mu \mathrm{g} / \mathrm{ml}$ and $10 \mu \mathrm{g} / \mathrm{ml}$ for $48 \mathrm{~h}$. Cells without 5-FU treatment were used as the negative control. Finally, $10 \mu \mathrm{lCCK}-8$ was added to each well. The OD values were measured at a wavelength of $450 \mathrm{~nm}$ (OD450). Cell survival was calculated as aforementioned.

Apoptosis assay. The cells were transfected with shRNA for $24 \mathrm{~h}$, then planted into 6 -well plates at a density of $1 \times 10^{6}$ cells per well. Subsequent to adhering to the wall, the cells were incubated with $4 \mu \mathrm{g} / \mathrm{ml} \mathrm{5-FU}$ for $24 \mathrm{~h}$. For the slide method, cells from each group were diluted twice with phosphate-buffered saline. Then, $5 \mu \mathrm{l}$ FITC and $5 \mu \mathrm{l}$ PI were added to $500 \mu \mathrm{l}$ binding buffer (Nanjing KeyGen Biotech Co., Ltd.), and the resulting mixture was added to the cell surface directly. Subsequent to incubation of the cells in the dark for 5-15 min at room temperature, the fluorescence was observed under the inverted fluorescence microscope. For flow cytometry, the cells were harvested and diluted twice with phosphate-buffered saline. Then, $5 \mu \mathrm{l}$ FITC and $5 \mu \mathrm{l}$ PI were added to $500 \mu \mathrm{l}$ of the cell suspension (final cell density, $\sim 6 \times 10^{5}$ cells $/ \mathrm{ml}$ ). Subsequent to incubation in the dark for 5-15 min at room temperature, flow cytometry was performed using a flow cytometer (FC500; Beckman Coulter, Inc., Brea, CA, USA). The experiments were performed independently three times.

Statistical analysis. All data were expressed as the mean \pm standard deviation, and SPSS 17.0 software (SPSS, Inc., Chicago, IL, USA) was used for statistical analyses. One-way analysis of variance and Student's $t$-test were used to analyze the significance 
A
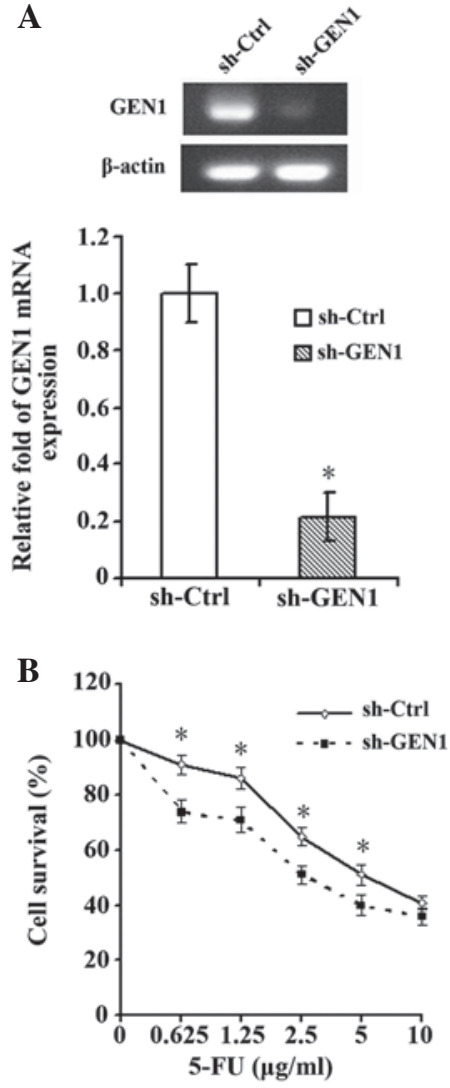

Figure 2. SKBR3 cells demonstrated increased sensitivity to 5-FU subsequent to suppression of the GEN1 gene. (A) The silencing of GEN1 in SKBR3 cells was confirmed by reverse transcription-quantitative polymerase chain reaction and western blot analysis. (B) Cells in the sh-Ctrl and sh-GEN1 groups were seeded onto 96-well culture plates at a density of 10,000 cells per well subesequent to $24 \mathrm{~h}$ of GEN1 suppression. The cell survival was determined using cell counting kit- 8 subsequent to treatment of cells with 5-FU for $48 \mathrm{~h}$. The results are expressed as the mean \pm standard deviation of triplicate samples from three experiments. The sh-GEN1 group demonstrated significantly enhanced sensitivity to 5-FU conpared with the sh-Ctrl group $(\mathrm{P}<0.05)$. (C) The cells were treated with the half maximal inhibitory concentration of 5-FU $(8.77 \mu \mathrm{g} / \mathrm{ml})$ and the survival of the cells in the sh-GEN1 group was significantly decreased compared with the cells in the sh-Ctrl group $(\mathrm{P}<0.05)$. "P<0.05, sh-Ctrl vs. sh-GEN1 group. 5-FU, 5-fluorouracil; GEN1, flap endonuclease GEN homolog 1; sh-Ctrl; control short hairpin RNA; sh-GEN1, GEN1 short hairpin RNA.

of differences between groups. Multiple comparisons between the groups was performed using the Student-Newman-Keuls method subsequent to one-way analysis of variance. $\mathrm{P}<0.05$ was considered to indicate a statistically significant difference.

\section{Results}

$I C_{50}$ values of 5-FU against SKBR3 and MCF-7 breast cancer cell lines. The survival curves of the SKBR3 and MCF-7 cells are shown in Fig. 1. The $\mathrm{IC}_{50}$ values of 5-FU against the breast cancer SKBR3 and MCF-7 cell lines were 8.77 and $7.73 \mu \mathrm{g} / \mathrm{ml}$, respectively, which were then used in subsequent experiments.

SKBR3 cells demonstrated increased sensitivity to 5-FU following GEN1 gene suppression. Silencing of the GEN1 gene was confirmed by RT-qPCR and western blot analysis (Fig. 2A). Following suppression of the GEN1 gene, the SKBR3 cells demonstrated significantly enhanced sensitivity to 5-FU. At 5-FU concentrations of $0.625,1.25,2.5$ and
A
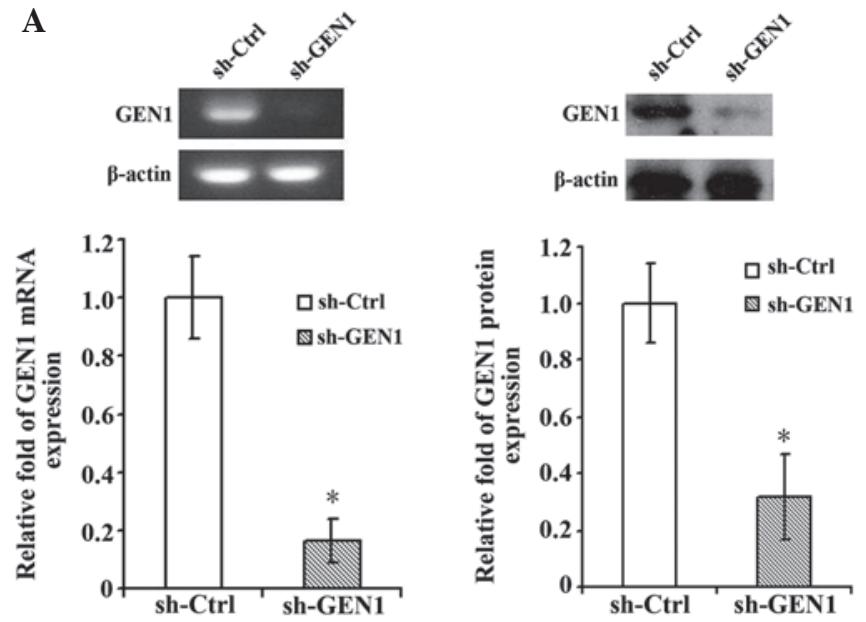

B

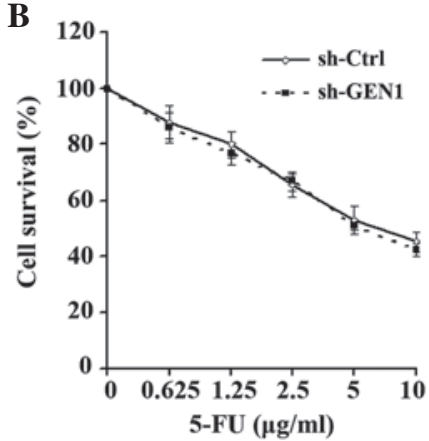

C

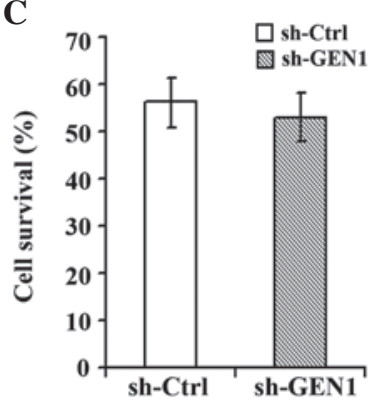

Figure 3. Silencing GEN1 in the MCF-7 cell line had no significant effect on the sensitivity to 5-FU. (A) The silencing of GEN1 in MCF-7 cells was confirmed by reverse transcription-quantitative polymerase chain reaction and western blot analysis. (B) Cells in the sh-Ctrl and sh-GEN1 groups were seeded onto 96-well culture plates at a density of 10,000 cells per well subsequent to $24 \mathrm{~h}$ of GEN1 suppression. The cell survival were determined using cell counting kit-8 subsequent to treatment of the cells with 5-FU for $48 \mathrm{~h}$. The results are expressed as the mean \pm standard deviation of triplicate samples from three experiments. Following GEN1 suppression, the MCF-7 cell line did exhibit a significant change in its sensitivity to 5-FU. (C) The cells were treated with the half maximal inhibitory concentration of 5-FU $(7.73 \mu \mathrm{g} / \mathrm{ml})$ and the survival rates of the two groups demonstrated no evident change. ${ }^{*} \mathrm{P}<0.05$, sh-Ctrl vs. sh-GEN1 group. 5-FU, 5-fluorouracil; GEN1, flap endonuclease GEN homolog 1; sh-Ctrl; control short hairpin RNA; sh-GEN1, GEN1 short hairpin RNA.

$5.0 \mu \mathrm{g} / \mathrm{ml}$, the survival of cells in the experimental sh-GEN1 group was significantly decreased compared with the control sh-Ctrl group $(\mathrm{P}<0.05$; Fig. $2 \mathrm{~B})$. When treated with the $\mathrm{IC}_{50}$ $(8.77 \mu \mathrm{g} / \mathrm{ml})$ of $5-\mathrm{FU}$, the survival of SKBR3 cells in the sh-GEN1 group was significantly reduced (Fig. 2C).

Silencing GEN1 in the MCF-7 cell line had no significant effect on the sensitivity to 5-FU. The silencing of GEN1 was confirmed by RT-qPCR and western blot (Fig. 3A). Subsequent to suppression of GEN1, the MCF-7 cell line did not demonstrate a significant change in the sensitivity to 5-FU. Under various concentrations of 5-FU, the survival of the sh-GEN1 group was similar with to the survival of the sh-Ctrl group (Fig. 3B). When treated with the $\mathrm{IC}_{50}$ of $5-\mathrm{FU}(7.73 \mu \mathrm{g} / \mathrm{ml})$, the survival rates of the sh-Ctrl and sh-GEN1 groups exhibited no evident difference in sensitivity (Fig. 3C). 
A
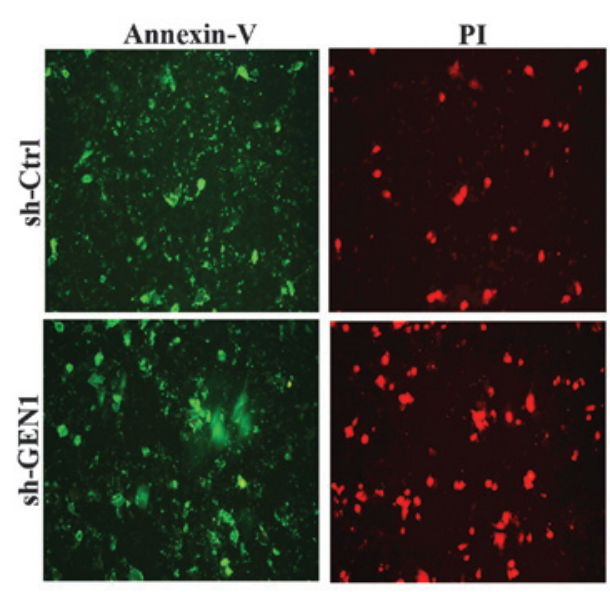

C
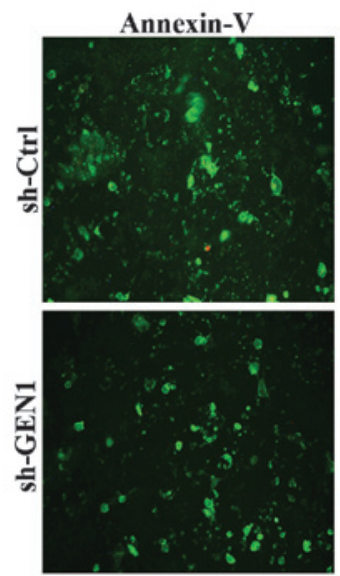

B
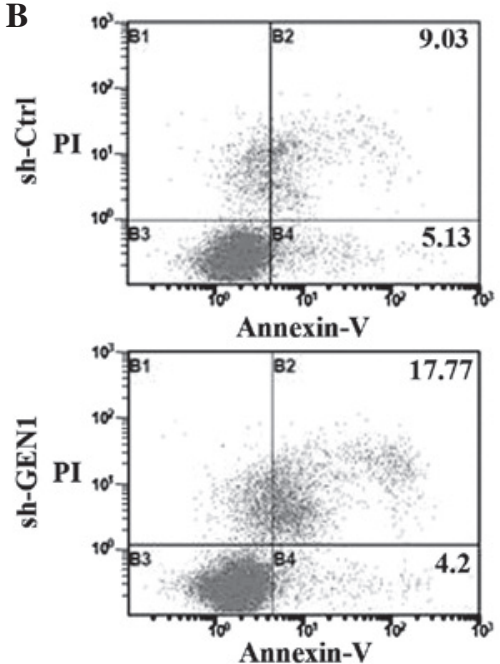

D
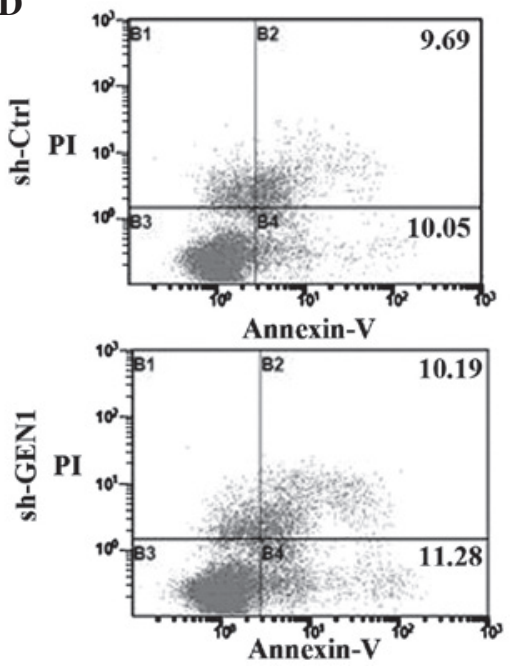

Figure 4. The effect of silencing GEN1 on apoptosis in SKBR3 and MCF-7 cells. (A) The apoptosis of SKBR3 cells was observed under a fluorescence microscope (magnification, x200). (B) The apoptosis of SKBR3 cells was detected by flow cytometry. (C) The apoptosis of MCF-7 was observed under a fluorescence microscope (magnification, x200). (D) The apoptosis of MCF-7 cells was detected by flow cytometry. GEN1, flap endonuclease GEN homolog 1; sh-Ctrl; control short hairpin RNA; sh-GEN1, GEN1 short hairpin RNA; PI, propidium iodide.

$24 \mathrm{~h}$, the cells were treated with 5-FU and the apoptosis was detected using fluorescence microscopy and flow cytometry. Under the fluorescence microscope, the fluorescence intensities of the SKBR3 cells of the sh-GEN1 group were significantly stronger $(\mathrm{P}<0.05)$ compared with the sh-Ctrl group (Fig. 4A), particularly red fluorescence. The result of flow cytometry also showed that the apoptosis of the sh-GEN1 group $(21.98 \pm 3.23 \%)$ was significantly increased $(\mathrm{P}<0.05)$ compared with the control group (14.76 $\pm 2.87 \%$ ) (Fig. 4B). However, in the MCF-7 cells, the results of each method did not demonstrate statistical differences between the two groups (Fig. 4C and D).

Suppression of the GEN1 and Mus81 genes increased the sensitivity of MCF-7 cells to 5-FU. The expression of the GEN1 and Mus81 genes was simultaneously suppressed to observe the effect on the sensitivity of MCF-7 cells to 5-FU. The silencing of the genes was confirmed by RT-qPCR and western blot analysis (Fig. 5A and B). The sh-Ctrl-1, sh-Ctrl-2, sh-GEN1, sh-Mus81 and sh-GEN1+sh-Mus81 groups were transfected with $4 \mu \mathrm{g}$ control plasmid, $8 \mu \mathrm{g}$ control plasmid,
$4 \mu \mathrm{g}$ sh-GEN1 plasmid, $4 \mu \mathrm{g}$ sh-Mus81 plasmid and $4 \mu \mathrm{g}$ sh-GEN1 plasmid $+4 \mu \mathrm{g}$ sh-Mus81 plasmid, respectively. No significant difference in cell survival was identified between the sh-Ctrl-1 and sh-Ctrl-2 groups and the sh-GEN1 group. Unlike GEN1 suppression alone, MCF-7 cells demonstrated significantly enhanced sensitivity to 5-FU when the GEN1 and Mus81 genes were simultaneously suppressed (Fig. 5C).

Under a low concentration of 5-FU, the GEN1 expression level increased when the Mus81 level was low. Subsequent to suppression of Mus81 for $24 \mathrm{~h}$, a low concentration of $1 \mu \mathrm{g} / \mathrm{ml}$ 5-FU, which was approximately one-quarter of the $\mathrm{IC}_{50}$ value, was added into the MCF-7 cells and the cells were incubated for 96 h. Following incubation, the expression levels of GEN1 and Mus81 were detected by RT-qPCR and western blot analysis. The results showed that the level of GEN1 in the experimental sh-Mus81 group was increased compared with the control sh-Ctrl group $(\mathrm{P}<0.05$; Fig. 6A). Additionally, the MCF-7 and SKBR3 cells of the experimental groups were incubated with $1 \mu \mathrm{g} / \mathrm{ml} 5$-FU for $96 \mathrm{~h}$ and cells cultured in normal medium 
A
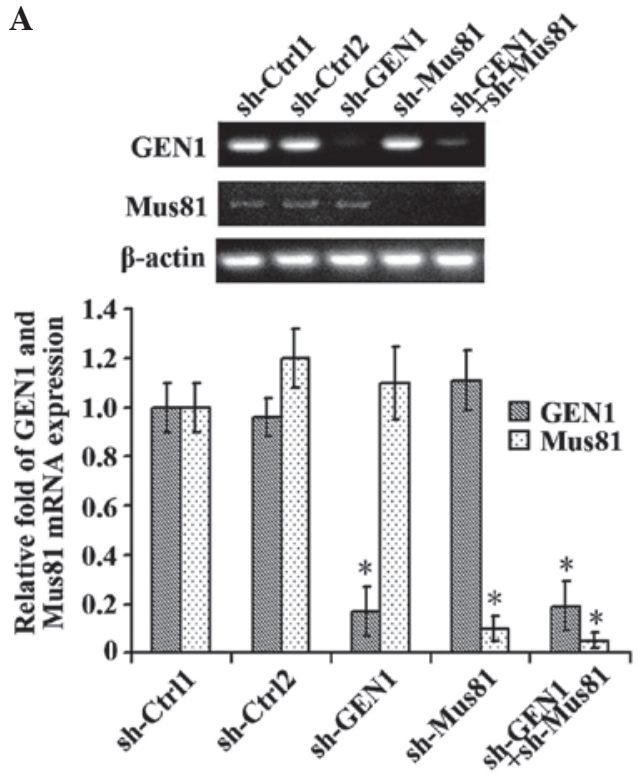

B
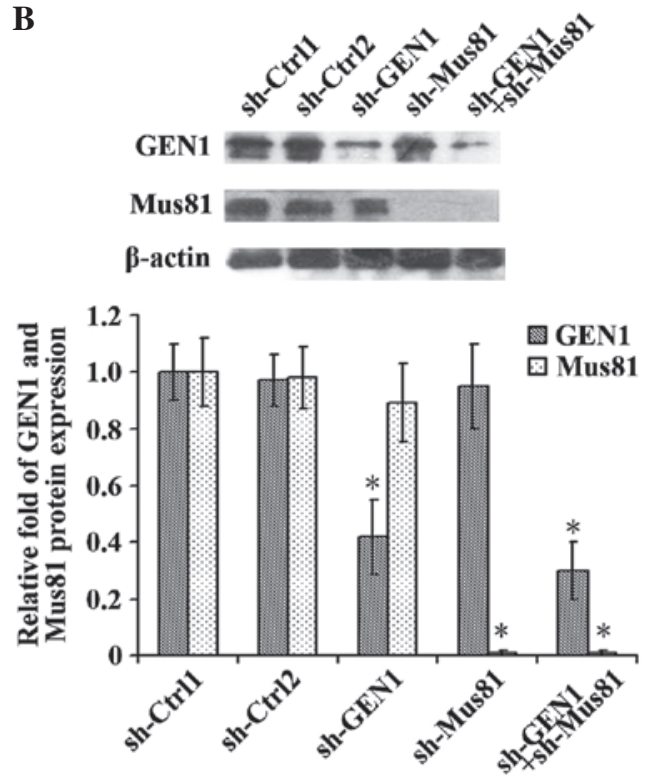

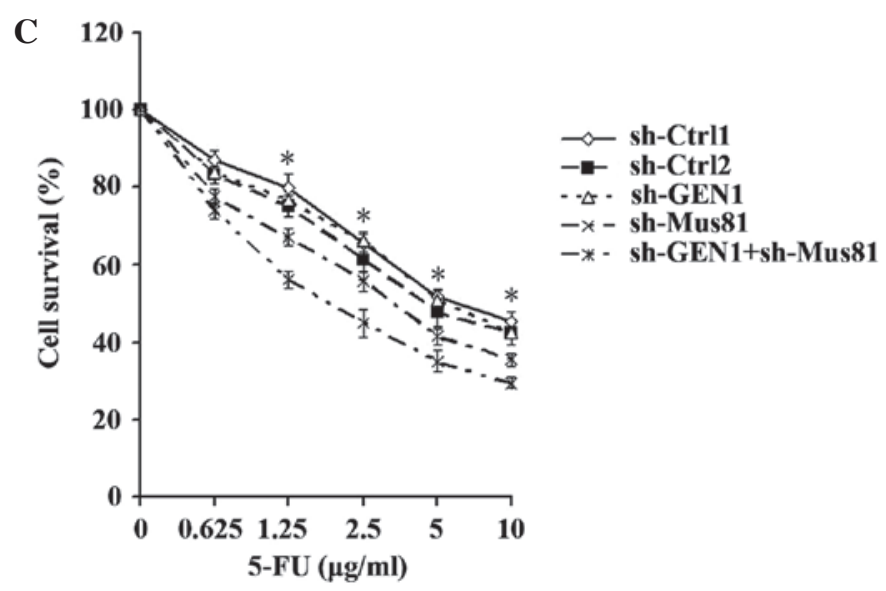

Figure 5. The MCF-7 cell line demonstrated increased sensitivity to 5-FU subsequent to the simultaneous suppression of the GEN1 and Mus81 genes. Reverse transcription-quantitative polymerase chain reaction and western blot analysis confirmed the silencing of the GEN1 and Mus 81 genes by assessing the (A) mRNA and (B) protein expression. (C) The sh-Ctrl-1, sh-Ctrl-2, sh-GEN1, sh-Mus81 and sh-GEN1+sh-Mus81 groups were transfected with $4 \mu$ g control plasmid, $8 \mu \mathrm{g}$ control plasmid, $4 \mu \mathrm{g}$ sh-GEN1 plasmid, $4 \mu \mathrm{g}$ sh-Mus81 plasmid and $4 \mu \mathrm{g}$ sh-GEN1 plasmid $+4 \mu \mathrm{g}$ sh-Mus81 plasmid, respectively. No significant difference in cell survival was identified between the sh-Ctrl-1 and sh-Ctrl-2 groups and the sh-GEN1 group. In contrast to the suppression of GEN1 alone, MCF-7 cells demonstrated significantly enhanced sensitivity to 5-FU when the GEN1 and Mus 81 genes were simultaneously suppressed (P<0.05). * $<0.05$. GEN1, flap endonuclease GEN homolog 1; Mus81, MUS81 structure-specific endonuclease subunit; sh-Ctrl; control short hairpin RNA; sh-GEN1, GEN1 short hairpin RNA; sh-Mus81, Mus81 short hairpin RNA; 5-FU, 5-fluorouracil.

were considered the control group. Subsequently, the expression level of GEN1 was detected in each group by western blot analysis (Fig. 6B). The GEN1 expression level in the MCF-7 cells, which demonstrated high Mus81 expression (Fig. 6C), did not change significantly when the cells were treated with 5-FU (Fig. 6Ba). However, compared with the control group, the level of GEN1 expression was increased in the SKBR3 cells $(\mathrm{P}<0.05$; Fig. 6Bb), which demonstrated a low Mus81 expression level (Fig. 6C).

\section{Discussion}

GEN1 was first identified in rice by Furukawa et al (3). Ishikawa et al classified GEN1 into a new class (class IV) of the Rad2/XPG nuclease family (4). GEN1 contains an N-terminal, internal conserved $\operatorname{Rad} 2 / \mathrm{XPG}$ endonuclease region and a helix-hairpin-helix region (17). Gao et al found that GEN1 functioned as an $\mathrm{HJ}$ resolvase in vivo as well as in vitro (18). In addition, a number of studies have confirmed that the HJ may be broken down by GEN1 $(19,20)$. Similar to the Escherichia coli $\mathrm{HJ}$ resolvase RuvC, GEN1 specifically binds and resolves HJs by a dual incision mechanism (17,21). GEN1 resolves $\mathrm{HJ}$ in a symmetrical manner and exists in two forms, consisting of the monomeric form and dimeric complexes. Efficient $\mathrm{HJ}$ resolution requires dimeric complexes in order to provide the two active sites required for near simultaneous dual incision (17). This monomer/dimer substrate-driven switch distinguishes GEN1 from other HJ resolvases, such as Mus81, which resolves HJ in an asymmetric manner (11).

HJs were first identified by Robin Holliday (22). Numerous studies hypothesized that HJ resolved properly is the key to correct DNA repair $(8,9)$. DNA damaging drugs, such as 5-FU, may injure tumor cells by damaging the DNA of the cells (23). During this process, numerous HJs may be produced $(24,25)$. 
A
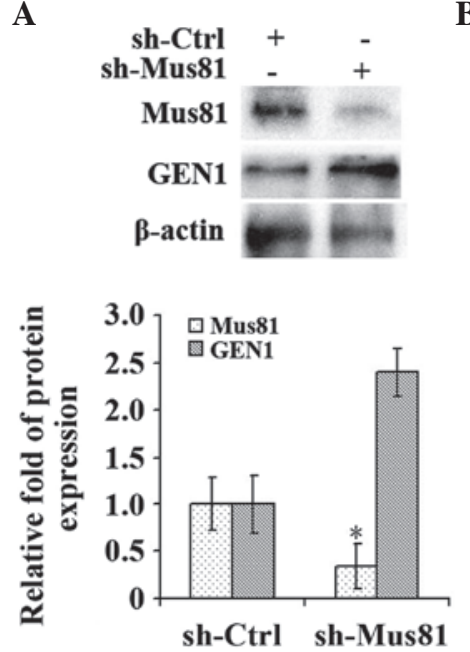

B

a
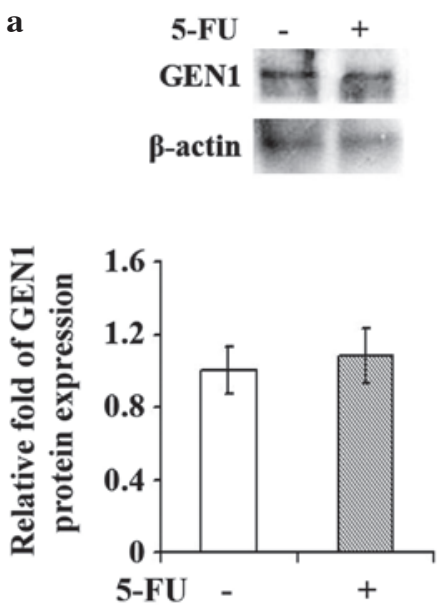

C b
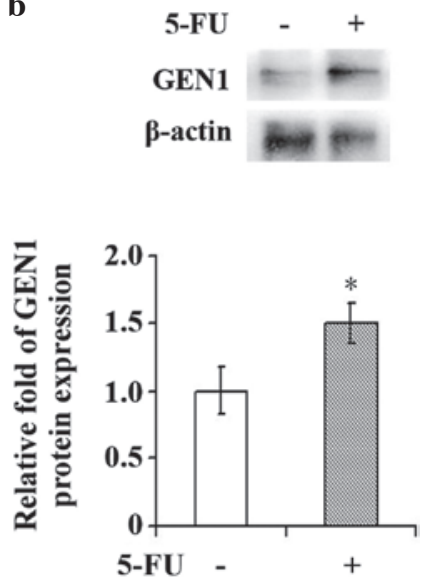
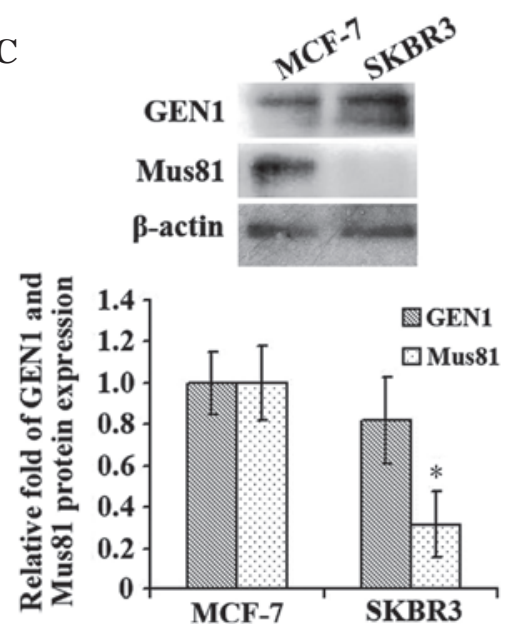

Figure 6. Under a low concentration of 5-FU, the GEN1 expression level increased when the Mus81 level was low. (A) The level of GEN1 in the MCF-7 cell line increased when Mus81 was suppressed and a low concentration of 5-FU was administered. (B) The level of GEN1 in the MCF-7 cell line did not change significantly when cells were treated with a low concentration of 5-FU. The GEN1 level in the SKBR3 cell line, however, increased significantly under the same conditions. (C) The expression levels of Mus81 in MCF-7 and SKBR3 cells without treatment. * $<<0.05$. GEN1, flap endonuclease GEN homolog 1; Mus81, MUS81 structure-specific endonuclease subunit; 5-FU, 5-fluorouracil.

It has been confirmed that subsequent to suppressing $\mathrm{HJ}$ resolvase-Slx4, the sensitivity to DNA damaging agents of tumor cells increased significantly. Previous studies have also confirmed that targeting Mus81 may increase sensitivity to DNA damaging drugs, such as 5-FU, in breast cancer cells (13). Thus, the HJ resolution capacity of tumor cells has a significant effect on the sensitivity to drugs. However, it remains unknown whether GEN1 may have an impact on the chemosensitivity of tumor cells. It has been proved that GEN1 interference may increase pharmaceutical sensitivity of yeast and Drosophila alone or in combination with other genes $(11,15)$. Therefore, the present study was designed to explore the effect of GEN1 interference on the chemosensitivity of breast cancer cell lines MCF-7 and SKBR3.

In the present study, the functional studies of GEN1 were investigated in MCF-7 and SKBR3 cells. shRNA was used to suppress the expression of GEN1 and the cells were incubated with 5-FU. Cell survival and apoptosis were detected to evaluate the effect of GEN1 expression on the two cell lines. The results showed that following suppression of GEN1, survival of SKBR3 cells in the experimental group was significantly decreased compared with the control group, while the level of apoptosis was significantly increased. These results suggested that the SKBR3 cell line increased the sensitivity to 5-FU following suppression of the GEN1 gene. By contrast, the MCF-7 cell line did not show significant changes under the same conditions, which indicated that targeting GEN1 had no evident effect on the chemosensitivity of MCF-7 cells. The aforementioned results demonstrated that there was a marked difference between the effect of GEN1 expression on the sensitivity to injury drugs in the two breast cell lines, which requires additional investigation. A previous study showed that the expression of Mus81 in the SKBR3 cells was decreased compared with MCF-7 cells (Fig. 6C). Since GEN1 and Mus81 did not resolve $\mathrm{HJ}$ in the same manner, with GEN1 adopting a symmetrical manner and Mus 81 using an asymmetric mode, the present study hypothesized that GEN1 acted as a collaborative gene of Mus81 and did not play a key role in breast cells with a high level of Mus81. However, the activity of GEN1 increases when the expression of Mus81 is low. To verify this hypothesis, the expression of GEN1 and Mus81 was suppressed simultaneously in the MCF-7 cell line. The results showed that although silencing GEN1 alone did not increase the sensitivity of MCF-7 cells to 5-FU, targeting GEN1 and Mus81 together significantly 
enhanced this sensitivity. Furthermore, the enhancement is also stronger than the effect of silencing Mus81 alone. Therefore, the possibility that this change was merely due to Mus81 interference was excluded, as the effect of GEN1 was also involved in the sensitivity of cells to 5-FU. However, no significant differences in the cell survival were identified between the sh-Ctrl-1 and sh-GEN1 groups, which indicated that GEN1 did not play a significant role in cell survival. The aforementioned results showed that GEN1 did not play a significant role in the presence of Mus81 and its effect will be reflected when Mus81 level was low. In addition, GEN1 interference enhanced the effect of Mus81 on the chemotherapy of breast cancer cells.

To further confirm the aforementioned statement, the MCF-7 cells were incubated with a low concentration of 5-FU for $96 \mathrm{~h}$ subsequent to Mus81 suppression. The level of GEN1 was determined by western blot analysis. The result showed that the GEN1 level in the experimental group was significantly increased compared with the control group. Therefore, when the SKBR3 cell line, which exhibited low Mus81 expression, was incubated with a low concentration of 5-FU, the GEN1 level in the experimental group also increased significantly. The aforementioned results indicated that GEN1 demonstrates enhanced function when the Mus81 level is low. By contrast, the normal MCF-7 cell line, which exhibited high Mus81 expression, did not demonstrate an enhanced GEN1 level when treated with 5-FU, which supports the present conclusion.

Overall, the present study showed that GEN1 may play different roles in diverse breast cancer cell lines. The function of GEN1 may be affected by the level of Mus 81 in the cell line. GEN1 acts as a collaborative gene of Mus81 and did not play a key role in the breast cell line with a high Mus81 level. The activity of GEN1 increases, however, in the cells with low expression of Mus81. In addition, it is worth mentioning that GEN1 interference improves the chemotherapy sensitizing effect brought by targeting Mus81 alone. If Mus81 alone is targeted over the course of breast cancer treatment, the expression level of GEN1 may increase gradually thus to reduce the treatment effect. Therefore, the present study hypothesizes that targeting Mus81 together with GEN1 may result in an improved effect of chemotherapy on breast cancer.

\section{Acknowledgements}

The present study was supported by the Natural Science Foundation of Zhejiang Province (grant no. Y14H200002), Medicines Health Platform Key Project of Zhejiang Province (grant no. 2013ZDA024) and Science and Technology Project of Shaoxing City (grant no. 2013D10040).

\section{References}

1. Torre LA, Bray F, Siegel RL, Ferlay J, Lortet-Tieulent J and Jemal A: Global cancer statistics, 2012. CA Cancer J Clin 65: 87-108, 2015.

2. Kuczynski EA, Sargent DJ, Grothey A and Kerbel RS: Drug rechallenge and treatment beyond progression - implications for drug resistance. Nat Rev Clin Oncol 10: 571-587, 2013.

3. Furukawa T, Kimura S, Ishibashi T, Mori Y, Hashimoto J and Sakaguchi K: OsSEND-1: A new RAD2 nuclease family member in higher plants. Plant Mol Biol 51: 59-70, 2003.

4. Kanai Y, Ishikawa G, Takeuchi R, Ruike T, Nakamura R, Ihara A, Ohashi T, Takata K, Kimura S and Sakaguchi K: DmGEN shows a flap endonuclease activity, cleaving the blocked-flap structure and model replication fork. FEBS J 274: 3914-3927, 2007.
5. Muñoz-Galván S, Tous C, Blanco MG, Schwartz EK, Ehmsen KT, West SC, Heyer WD and Aguilera A: Distinct roles of Mus81, Yen1, Slx1-Slx4 and Rad1 nucleases in the repair of replication-born double-strand breaks by sister chromatid exchange. Mol Cell Biol 32: 1592-1603, 2012.

6. Agmon N, Yovel M, Harari Y, Liefshitz B and Kupiec M: The role of Holliday junction resolvases in the repair of spontaneous and induced DNA damage. Nucleic Acids Res 39: 7009-7019, 2011.

7. Ip SC, Rass U, Blanco MG, Flynn HR, Skehel JM and West SC: Identification of Holliday junction resolvases from humans and yeast. Nature 456: 357-361, 2008.

8. Ashton TM, Mankouri HW, Heidenblut A, McHugh PJ and Hickson ID: Pathways for Holliday junction processing during homologous recombination in saccharomyces cerevisiae. Mol Cell Biol 31: 1921-1933, 2011.

9. Machwe A, Karale R, Xu X, Liu Y and Orren DK: The Werner and Bloom syndrome proteins help resolve replication blockage by converting (regressed) holliday junctions to functional replication forks. Biochemistry 50: 6774-6788, 2011.

10. Saito TT, Mohideen F, Meyer K, Harper JW and Colaiácovo MP: SLX-1 is required for maintaining genomic integrity and promoting meiotic noncrossovers in the Caenorhabditis elegans germline. PLoS Genet 8: e1002888, 2012.

11. Andersen SL, Kuo HK, Savukoski D, Brodsky MH and Sekelsky J: Three structure-selective endonucleases are essential in the absence of BLM helicase in Drosophila. PLoS Genet 7: e1002315, 2011.

12. Svendsen JM, Smogorzewska A, Sowa ME, O'Connell BC, Gygi SP, Elledge SJ and Harper JW: Mammalian BTBD12/SLX4 assembles a Holliday junction resolvase and is required for DNA repair. Cell 138: 63-77, 2009.

13. Qian Y, Liu Y, Yan Q, Lv J, Ni X, Wu Y and Dong X: Inhibition of Mus81 by siRNA enhances sensitivity to 5 -FU in breast carcinoma cell lines. Onco Targets Ther 7: 1883-1890, 2014.

14. Vizeacoumar FJ, Arnold R, Vizeacoumar FS, Chandrashekhar M, Buzina A, Young JT, Kwan JH, Sayad A, Mero P, Lawo S, et al: A negative genetic interaction map in isogenic cancer cell lines reveals cancer cell vulnerabilities. Mol Syst Biol 9: 696, 2013.

15. Ho CK, Mazón G, Lam AF and Symington LS: Mus81 and Yen1 promote reciprocal exchange during mitotic recombination to maintain genome integrity in budding yeast. Mol Cell 40: 988-1000, 2010.

16. Wechsler T, Newman S and West SC: Aberrant chromosome morphology in human cells defective for Holliday junction resolution. Nature 471: 642-646, 2011.

17. Rass U, Compton SA, Matos J, Singleton MR, Ip SC, Blanco MG, Griffith JD and West SC: Mechanism of Holliday junction resolution by the human GEN1 protein. Genes Dev 24: 1559-1569, 2010.

18. Gao M, Rendtlew Danielsen J, Wei LZ, Zhou DP, Xu Q, Li MM, Wang ZQ, Tong WM and Yang YG: A novel role of human holliday junction resolvase GEN1 in the maintenance of centrosome integrity. PLoS One 7: e49687, 2012.

19. Bailly AP, Freeman A, Hall J, Déclais AC, Alpi A, Lilley DM, Ahmed S and Gartner A: The caenorhabditis elegans homolog of Gen1/Yen1 resolvases links DNA damage signaling to DNA double-strand break repair. PLoS Genet 6: e1001025, 2010.

20. Tay YD and Wu L: Overlapping roles for Yen1 and Mus81 in cellular Holliday junction processing. J Biol Chem 285: 11427-11432, 2010.

21. Górecka KM, Komorowska W and Nowotny M: Crystal structure of RuvC resolvase in complex with Holliday junction substrate. Nucleic Acids Res 41: 9945-9955, 2013.

22. Holliday R: A mechanism for gene conversion in fungi. Genet Res 5: 282-304, 1964.

23. Tetu SG, Johnson DA, Varkey D, Phillippy K, Stuart RK, Dupont CL, Hassan KA, Palenik B and Paulsen IT: Impact of DNA damaging agents on genome-wide transcriptional profiles in two marine Synechococcus species. Front Microbiol 16: 232, 2013.

24. Lorenz A, West SC and Whitby MC: The human Holliday junction resolvase GEN1 rescues the meiotic phenotype of a Schizosaccharomyces pombe mus 81 mutant. Nucleic Acids Res 38: 1866-1873, 2010.

25. Mankouri HW, Ashton TM and Hickson ID: Holliday junction-containing DNA structures persist in cells lacking Sgs1 or Top3 following exposure to DNA damage. Proc Natl Acad Sci USA 108: 4944-4949, 2011. 